\title{
ADDITIONAL EXTREME DISTRIBUTION FOR MODELING EXTREME VALUE DATA
}

\author{
O. M. Khaled and Aya Kamal \\ Dep. of Mathematics, Faculty of Science, \\ Port Said University, Egypt
}

Received 17/2/2017

Revised 29/7/2017

Accepted 3/8/2017

\begin{abstract}
In this article an additional extreme value distribution (AEVD) is introduced by Box-Cox transformation. Both generalized Pareto distribution under power normalization (GPDP) and generalized extreme value distribution under power normalization (GEVP) can be obtained from AEVD as special cases. The AEVD can be applied in a wide variety of application fields especially for modelling extreme data. A system of nonlinear equations is solved numerically to get maximum likelihood estimation (MLE) for the distribution parameters. The proposed distribution is applied for modelling daily maximum air pollution from Barking Dagenham station. Various criteria reveal that AEVD improves the fitting of this data compared with other distributions.
\end{abstract}

Subject Classifications: 62G32, 60G70, 62F40.

Key words: Generalized extreme value distribution; Box-Cox-GEVP distribution; Air Pollution.

\section{Introduction}

Probabilistic extreme value theory is a curious and enchanting blend of an tremendous variety of applications including natural phenomena such as floods, rainfall, air pollution, corrosion and wind gusts. First of all, probabilistic extreme value theory deals with the asymptotic behaviour of the maximum and the minimum order statistics of identically distribution (iid) random variables (rv's). The distributional properties of extremes and intermediate order statistics, and exceedances over (below) high (low) thresholds are strongly depend on the upper and lower tails of the underlying distribution.

Extreme value distributions (EVDs) originally appeared as three possible limiting distributions of suitably linear normalization of a sequence of iid rv's. EVDs are the foundation of modelling and analyzing rare values, especially in environmental data e.g. daily maximum ozone measurements, maximum wind speed and and maximum water level. Generally, two well known methods, Block Maxima (BM) and Peak Over Threshold (POT) are used to analyze such data. The BM metod modeled by generalized extreme value distribution under linear normalization (GEVL) was introduced by [1]. On the other hand, Peak Over Threshold (POT) modeled by generalized Pareto distribution under linear normalization (GPDL) was proposed by [2]. A more general extreme value distribution named Box-Cox-GEVL was introduced by [3] and it is applied for stock data. However, some of previous distributions are not adequate in many areas. Two alternative models are built via some of the possible limiting distributions of power normalized maxima obtained by Pantcheva. The first probability model in this context, is GEVP which was discussed in [4] while the second one was introduced by [6] and it is known as GPDP. [5] applied BM method to analyze air pollutants SO2, PM10 and Ozone data using GEVP. Recently, [7] suggested and studied a new model, linear-power model to overcome some drarback of the power model In this paper, we give a simple generalization of $\mathrm{BM}$ and POT methods through the application of AEVD to model air pollutant data from Barking Dagenham station during the period 1-1-2010 to 31-12-2015, for daily maximum nitrogen oxides, nitrogen dioxide and sulfur dioxide concentrations. 


\section{Preliminaries}

\subsection{Box-Cox distribution under linear normalization}

Let $Y_{1}, Y_{2}, \cdots, Y_{n}$ be iidrv's with distribution function (df) $F(y)=P(Y \leq y)$ and assume $Y_{1: n}<Y_{2: n}<\cdots<Y_{n: n}$ be the corresponding order statistics. [1] re-parameterized the Extreme type Theorem for modeling the rare event. We can summarize this theorem as: for any constant in sequences $c_{n}>0$ and $d_{n}$ if $P\left(Y_{n: n} \leq c_{n} y+d_{n}\right)=F^{n}\left(c_{n} y+d_{n}\right)$ weakly converged to a nondegenerate df $L(x)$, then $L$ ought to be of a similar type of the GEVL

$$
L_{\gamma}(y ; \mu, \sigma)=\exp \left[-\left[1+\gamma\left(\frac{y-\mu}{\sigma}\right)\right]^{-\frac{1}{\gamma}}\right] 1+\gamma(y-\mu) / \sigma>0,
$$

which is a unique model for the extreme value distribution, where $\mu$ and $\sigma>0$ are location and scale parameters. The GEVL produces three different distributions, according to $\gamma<0 \gamma>0$, and $\gamma=0(\gamma \rightarrow 0)$, which are known as Weibull, Frechet and Gumbel families of df's, respectively. So, many statistical method either parametric or nonparametric derived from the theory of estimation can be used to estimate the parameters $\gamma, \sigma$ and $\mu$.

The BM approach is applied when the data is independent sample maxima. In fact, some of these blocks contain small or nil values whereas others may contain extremely large values. So, many important data are lost, and consequently the BM method becomes unsuitable for the few data. The above reason restricts the utilization of the BM method . Therefore, the Peak Over Threshold ( POT) method is considered an another trend for extreme data modeling through asymptotic distribution, initiated by [2]. The GPDL is the limit distribution of scaled peaks exceeding high thresholds $y>\mu$, which can be written as,

$$
H_{\gamma}(y ; \mu, \sigma)=1-\left[1+\gamma\left(\frac{y-\mu}{\sigma}\right)\right]^{-\frac{1}{\gamma}} .
$$

When $\gamma<0$ there is an upper end point, so $\mu<y \leq \mu-\frac{\sigma}{\gamma}$. The GPDL family implies the exponential, uniform or Pareto distributions based on $\gamma$ value. Evidently, in the statistical modeling of POT method all data is used, rather than using the BM method.

[3] used the Boc-Cox transformation (see also, [8]) to composite GEVL and GPDL in the following distribution

$$
W_{\gamma}(x ; \mu, \sigma, \lambda)=\left[\frac{\exp \left[-\lambda\left[1+\gamma\left(\frac{y-\mu}{\sigma}\right)\right]^{-\frac{1}{\gamma}}\right]-1}{\lambda}\right]+1,
$$

with probability density function

$$
w_{\gamma}(x ; \mu, \sigma, \lambda)=\frac{1}{\sigma} \exp \left[-\lambda\left[1+\gamma\left(\frac{y-\mu}{\sigma}\right)\right]^{-\frac{1}{\gamma}}\right]\left[1+\gamma\left(\frac{x-\mu}{\sigma}\right)\right]^{-\frac{1}{\gamma}-1},
$$

and inverse distribution

$$
W_{\gamma}^{-1}(x ; \mu, \sigma, \lambda)=\mu-\frac{\sigma}{\gamma}+\frac{\sigma}{\gamma}\left(-\frac{1}{\lambda} \log (\lambda(q-1)+1)\right)^{-\gamma} .
$$

The distribution introduced in (2.3) is called Box-Cox-GEVL, which tends to the GPDL and GEVL distribution. Specifically, when $\lambda=1, W_{\gamma}(y) \rightarrow L_{\gamma}(y)$, and when $\lambda=0, W_{\gamma}(y) \rightarrow H_{\gamma}(y)$ by L'Hopital's Method. To get the support of distribution defined in (2.3), first when $\lambda \neq 0$ and $\gamma>0$ we get unbounded upper tail. To find the Lower bound let $R(y)=\exp \left(-\lambda\left(1+\gamma\left(\frac{y-\mu}{\sigma}\right)\right)^{-\frac{1}{\gamma}}\right)$. Then

$$
\begin{gathered}
\frac{R(y)-1}{\lambda}+1 \geq 0 \text { i.e., } \\
\exp \left(-\lambda\left(1+\gamma\left(\frac{y-\mu}{\sigma}\right)\right)^{-\frac{1}{\gamma}}\right) \geq 1-\lambda, \text { i.e., } \\
y \geq \mu+\frac{\sigma}{\gamma}\left(\left(\frac{-\lambda}{\log (1-\lambda)}\right)^{\gamma}-1\right) .
\end{gathered}
$$


Therefore, when $\lambda=0$, we get the lower support $x>\mu$

On the other hand, when $\lambda \neq 0$ and $\gamma<0$, to find the upper bound let $-\gamma=\gamma^{*}$. Then $\frac{R(y)-1}{\lambda}+1 \leq 1$, which implies that $y \leq \mu-\frac{\sigma}{\gamma^{*}}$. For the lower bound, for which $\frac{R(y)-1}{\lambda}+1 \geq 0$, then $x \geq \mu+\frac{\sigma}{\gamma^{*}}\left(1-\left(\frac{-\log (1-\lambda)}{\lambda}\right) \gamma^{*}\right)$, when $\lambda=0$ we get the boundary of GPDL as $\mu<x \leq \mu-\frac{\sigma}{\gamma}$.

\subsection{The Box-Cox- under Power Normlization Distribution}

In 1984 Pancheva began to develop the theory of extreme values under power normalization and then [9] showed that the p-max stable dfs attract more dfs than linear max stable $\mathrm{dfs}$, which practically means that the linear model may fail to model the data, while the power model succeed to do that, but not the vice versa. [10] considered the power normalization and derived all the possible limit df's $H$ of $Y_{n: n}=\max \left(Y_{1}, \ldots, Y_{n}\right)$ subjected to this normalization. Namely, [10] determined all the possible limit types of $H$, for which

$$
F^{n}\left(c_{n}|y|^{d_{n}} \mathcal{S}(x)\right)=P\left(C_{n}^{-1}\left(Y_{n: n}\right) \leq x\right)=P\left[\left|\frac{Y_{n: n}}{a_{n}}\right|^{\frac{1}{b_{n}}} \mathcal{S}\left(X_{n: n}\right) \leq x\right] \stackrel{w}{\longrightarrow} H(y),
$$

where $a_{n}>0$ and $b_{n}>0$ are reasonable power normalizing constants and $\mathcal{S}(y)=-1,0,1$, if $y<0, y=0, y>0$, respectively. These limit types are usually called the power max stable df's ( $p$-max stable df's). The $p-\max$ stable df's of six $p$-types of df's are $V_{1 ; \xi}(y)=\exp \left(-(\log y)^{-\xi}\right), y>1 ; V_{2 ; \xi}(y)=\exp \left(-(-\log y)^{\xi}\right), 0<y \leq 1 ; V_{3 ; \xi}(y)=\exp \left(-(-\log (-y))^{-\xi}\right)$, $-1<y \leq 0 ; V_{4 ; \xi}(y)=\exp \left(-(\log (-y))^{\xi}\right), y \leq-1 ; V_{5}(y)=\exp \left(-\frac{1}{y}\right), y>0 ;$ and $V_{6}(y)=\exp (-|y|), y \leq 0$.

Moreover, [11] stated that these $p$-types attract more than linear stable df's. Therefore, using the power normalization allow to obtain a wider class of limit df's, which can be used in solving approximation problems. Consequently, one can basically expand the area of applications of the extreme value model. A merged approach to the results of [9] and [11] has been obtained by [12]. As in the case of nonlinear normalization, [4] has summarized these types by the following von Mises type representations

$$
P_{1 ; \gamma}(y ; a, b)=\exp \left[-\left(1+\gamma \log a y^{b}\right)^{-\frac{1}{\gamma}}\right], y>0,1+\gamma \log a y^{b}>0,
$$

and

$$
P_{2 ; \gamma}(y ; a, b)=\exp \left[-\left(1-\gamma \log a(-y)^{b}\right)^{-\frac{1}{\gamma}}\right], y<0,1-\gamma \log a(-y)^{b}<0 .
$$

[6] introduced GPDP for each of the models (3.2) and (3.3), by $Q_{1 ; \gamma}(y)=1+\log P_{1 ; \gamma}(y ; a, b)$ and $Q_{2 ; \gamma}(y)=1+$ $\log P_{2 ; \gamma}(y ; a, b)$, respectively. It can be shown that (c.f., Theorem 2.2, [6]) each of the GPDP satisfies the POT stability property, i.e., the left truncated GPDP yields a GPDP again .

The block method are modeled by equation (3.2) for positive data and the corresponding model for POT method can be written as the following

$$
Q_{1 ; \gamma}(y ; a, b)=1-\left(1+\gamma \log a y^{b}\right)^{-\frac{1}{\gamma}}, y \geq\left(\frac{1}{a}\right)^{\left(\frac{1}{b}\right)} .
$$

When $\gamma<0$ there is a finite support $\left(\frac{1}{a}\right)^{\left(\frac{1}{b}\right)} \leq x \leq\left(\frac{1}{a} \exp \left(\frac{1}{|\gamma|}\right)\right)^{\frac{1}{b}}$. We propose more general extreme value distribution under nonlinear normlization constant using Box and Cox transformation in the following distribution

$$
F_{1, \gamma}(y ; a, b, \lambda)=\left[\frac{\exp \left[-\lambda\left(1+\gamma \log a y^{b}\right)^{-\frac{1}{\gamma}}\right]-1}{\lambda}\right]+1
$$

with probability density function

$$
f_{1, \gamma}(y ; a, b, \lambda)=\frac{b}{y} \exp \left[-\lambda\left(1+\gamma \log a y^{b}\right)^{-\frac{1}{\gamma}}\right]\left(1+\gamma \log a y^{b}\right)^{-\frac{1}{\gamma}-1},
$$

and inverse function

$$
F_{1, \gamma}^{-1}(y ; a, b, \lambda)=\exp \left[\left(-\frac{1}{\lambda} \log (\lambda(q-1)+1)\right)^{-\gamma}-\frac{1}{b \gamma}-\frac{\log (a)}{b}\right] .
$$

The Box-Cox-GEVP1 distribution introduced in Eq. (3.5) tend to the GPDP of [6] in Eq. (3.4) and the GEVP distribution of [4] for positive data. Specifically, when $\lambda=1$ the Box-Cox-GEVP1 reduces to the GEVP distribution. When $\lambda=$ 
0 equals zero, the Box.Cox.GEV converges to the GPDP for positive data by L'Hopital's method. To get the support defined of distribution defined in (3.5), first when $\lambda \neq 0$ and $\gamma>0$, we get unbounded upper tail. To find the lower bound, let $J(y)=\exp \left(-\lambda\left(1+\gamma \log a y^{b}\right)^{-\frac{1}{\gamma}}\right.$ then the lower bounded is given from the inequality $\frac{J(y)-1}{\lambda}+1 \geq 0$, i.e. $y \geq\left(\frac{1}{a} \exp \left(\frac{\left(\frac{-\lambda}{\log (1-\lambda}\right)^{\gamma}}{\gamma}-\frac{1}{\gamma}\right)\right)^{\frac{1}{b}}$, when $\lambda=0$ we get the boundary of GPDP as $y \geq\left(\frac{1}{a}\right)^{\left(\frac{1}{b}\right)}$. Second, when $\lambda \neq 0$ and $\gamma<0$, to find the upper bound, let $-\gamma=\gamma^{*}$ and $\frac{J(y)-1}{\lambda}+1 \leq 1$ then $y \leq\left(\frac{1}{a} \exp \left(\frac{1}{\gamma^{*}}\right)\right)^{\frac{1}{b}}$ For lower bound let $\frac{J(y)-1}{\lambda}+1 \geq 0$ then $y \geq\left(\frac{1}{a} \exp \left(\frac{-\left(\frac{-\log (1-\lambda}{\lambda}\right)^{*}}{\gamma^{*}}+\frac{1}{\gamma^{*}}\right)^{\frac{1}{b}}\right.$ when $\lambda=0$ we tend to the boundary of $\operatorname{GPDP}\left(\frac{1}{a}\right)^{\left(\frac{1}{b}\right)} \leq y \leq\left(\frac{1}{a} \exp \left(\frac{1}{|\gamma|}\right)\right)^{\frac{1}{b}}$

Also, we can get Box-Cox-GEVP2 for negative data by BMP method by (3.3) and POTP method by

$$
Q_{2 ; \gamma}(y ; a, b)=1-\left(1-\gamma \log a(-y)^{b}\right)^{-\frac{1}{\gamma}},
$$

When $\gamma>0$ there is upper and lower bounded, so $\left(\frac{1}{a}\right)^{\left(\frac{1}{b}\right)} \leq-y \leq\left(\frac{1}{a} \exp \left(\frac{1}{\gamma}\right)\right)^{\left(\frac{1}{b}\right)}$, Similarly when $\gamma<0$, let $-\gamma=\gamma^{*}$, $\left(\frac{1}{a} \exp \left(\frac{-1}{\gamma^{*}}\right)\right)^{\left(\frac{1}{b}\right)} \leq-y \leq\left(\frac{1}{a}\right)^{\left(\frac{1}{b}\right)}$.

We can summrize the two equation (3.3) and (3.6) in the following distribution

$$
F_{2, \gamma}(y ; a, b, \lambda)=\left[\frac{\exp \left[-\lambda\left(1-\gamma \log a(-y)^{b}\right)^{-\frac{1}{\gamma}}\right]-1}{\lambda}\right]+1
$$

We can also get the probability density function and inverse function for this distribution as before. This distribution nested to GEVP in Eq. (3.3), when $\lambda=1$ and GPDP in Eq. (3.6) when $\lambda=0$. For application model (3.7) for real data we must known the upper and lower bounded of this model, which is given by: when, $\lambda \neq 0$ and $\gamma>0$ thus,

$$
\begin{aligned}
& \left(\frac{\exp \left(\frac{-\left(\frac{-\log (1-\lambda)}{\lambda}\right)-\gamma-1}{\gamma}\right)}{a}\right)^{\frac{1}{b}} \leq-y \leq\left(\frac{\exp \left(\frac{1}{\gamma}\right)}{a}\right)^{\frac{1}{b}} \text { and when } \lambda \neq 0 \text { and } \gamma<0 \text { consequently, let }-\gamma=\gamma^{*} \text {, we get }\left(\frac{\exp \left(\frac{-1}{\gamma^{*}}\right)}{a}\right)^{\frac{1}{b}} \leq \\
& -y \leq\left(\frac{\exp \left(\frac{\left(\frac{-\log (1-\lambda)}{\lambda}\right)^{*}-1}{\gamma^{*}}\right)}{a}\right)^{\frac{1}{b}}
\end{aligned}
$$

\section{Air Pollution Application}

In this section we apply the new suggested models on a data set for air pollution from the London Air Quality Network (LAQN). This network is unified resource for air pollution measurements which are fundamental to support air quality administration. The LAQN was framed in 1993 to arrange and enhance air pollution checking in London. People living in London city can check the updates of the level of air pollution data through the web site www.londonair.org.uk. The greater part of London's 33 districts supply estimations to the system and also these information are progressively being supplemented by estimations from neighborhood specialists encompassing London, in this manner giving a general point of view of air pollution in London and the Home Counties. [13] clear that, All measurements under go quality control and assurance procedures with each measurement traceable to national metrological standards provided by the National Physical Laboratory . [14] developed empirical trend to predict the annual mean of $\mathrm{NO}_{2}$ levels in London using observation data. [15] analyzed the relationship between air contaminations and ischemic stroke subtypes. In this study data was taken from site Barking Dagenham at Rush Green square, that monitors Nitrogen oxides, sulphur dioxide, PM10 and climatology data. The daily maximum level of the nitric oxide $(\mathrm{NO})$, nitrogen dioxide $\left(\mathrm{NO}_{2}\right)$ and sulphur dioxide $\left(\mathrm{SO}_{2}\right)$ were recorded every hour. So a total of 53000 readings have been obtained in the period from 1-1-2010 to 31-12-2015. This data can be downloaded by any researcher in the form of a report every half hour, every hour or every day according to the type of study from the following site www.londonair.org.uk/london/asp/datadownload.asp. The daily maximum of these data was used for application of these models. The summary statistics is given in the Table 1 .

In this case study we deal with this data as follows: first we estimate the parameter of block data (daily maximum) by GEVL model and estimate the peak over mean of the data using GPDL model by MLE method. The log-likelihood of 
Table 1: Descriptive statistics for maximum data for air pollution

$\|$\begin{tabular}{|c|c|c|c|c|c|c|c|c}
\hline & $\mathrm{n}$ & minimum & maximum & median & mean & SD & skewness & kurtosis \\
\hline $\mathrm{NO}$ & 2006 & 0.2 & 597.7 & 10.7 & 31.29 & 51.67776 & 3.65 & 19.570 \\
\hline $\mathrm{NO}_{2}$ & 2003 & 2.30 & 229.1 & 45.1 & 47.417 & 22.18 & 0.935 & 2.814 \\
\hline $\mathrm{SO}_{2}$ & 2077 & 0.1 & 67.3 & 4.1 & 5.013 & 4.16 & 7.57 & 89.02 \\
\hline
\end{tabular}

Box-Cox GEVL distribution is:

$$
\ell_{\gamma}(y ; \mu, \sigma, \lambda)=-k \log \sigma-\lambda \sum_{i=1}^{k}\left[1+\gamma\left(\frac{y_{i}-\mu}{\sigma}\right)\right]^{-\frac{1}{\gamma}}-\left(1+\frac{1}{\gamma}\right) \sum_{i=1}^{k} \log \left[1+\gamma\left(\frac{y_{i}-\mu}{\sigma}\right)\right]
$$

Eq (3.8) gives the log-likelihood for GEVL distribution if $\lambda=1$, and GPDl distribution if $\lambda=0$. Analytically we cannot maximize the log-likelihood function in $\mathrm{Eq}$ (3.8), so we get the negative log-likelihood for minimization by using FindMinimum function in mathematica Package. Table 2 summarizes the MLE results, including parameter estimates, Akaike Information Criteria (AIC) and Bayesian Akaike Criteria (BIC), for comparison the models, which have small values of AIC and BIC are more favorable. ALso, we used Kolmogorov-Smirnov (K-S) test, where in this test we have four parameters $[h, p, K, c . v]$. Namely, $h$ is equal to 0 or $1, p$ is the $p$-value, $K$ is the maximum difference between fitting curve and the empirical distribution and c. $v$ is a critical value. Therefore, the null hypotheses is rejected when $h=1$ and $K>c . v$ and accepted when $h=0$, and $K \leq c . v$

Table 2: The MLE results, including parameter estimates, (AIC) and (BIC)

\begin{tabular}{||c|c|c|c|c|c||}
\hline \multicolumn{6}{|c|}{ Estimation parameter for maximum data by using GEVL model } \\
\hline Pollutant & $\gamma$ & $\sigma$ & $\mu$ & $\mathrm{AIC}$ & $\mathrm{BIC}$ \\
\hline $\mathrm{NO}$ & 1.075 & 7.907 & 6.856 & 8518.99 & 17044.8 \\
\hline $\mathrm{NO}_{2}$ & -0.0396 & 18.3 & 37.53 & 8944.9 & 17900 \\
\hline $\mathrm{SO}_{2}$ & 0.1806 & 1.6008 & 3.634 & 4473.51 & 8957.94 \\
\hline Estimation parameter for peak over threshold data by GPDL model \\
\hline Pollutant & $\gamma$ & $\sigma$ & $\mu$ (thrushold) & AIC & BIC \\
\hline $\mathrm{NO}$ & 0.1634 & 50.32 & 31.227 & 2761.08 & 5529.04 \\
\hline $\mathrm{NO}_{2}$ & -0.07077 & 20.34 & 47.4170 & 3677.32 & 7363.14 \\
\hline $\mathrm{SO}_{2}$ & 0.396 & 1.996 & 5.0138 & 1351.04 & 2709.48 \\
\hline
\end{tabular}

Table 3: K-S test

$\|$\begin{tabular}{c|c|c|c|c|c}
\hline \multicolumn{6}{c}{ Fitting parameter for GEVL } \\
\hline Pollutant & $h$ & $p$ & $K$ & $c . v$ & Decision \\
\hline $\mathrm{NO}$ & 1 & 0.06 & 0.032 & 0.0272 & reject model \\
$\mathrm{NO}_{2}$ & 0 & 0.1662 & 0.0211 & 0.0273 & accepted model \\
$\mathrm{SO}_{2}$ & 1 & $7.99 * 10^{-7}$ & 0.0245 & 0.0636 & reject model \\
\hline \multicolumn{6}{|c}{ Fitting parameter for GPDL } \\
\hline Pollutant & $h$ & $p$ & $K$ & $c . v$ & Decision \\
\hline $\mathrm{NO}$ & 1 & 0 & 0.2916 & 0.0272 & reject model \\
$\mathrm{NO}_{2}$ & 1 & 0 & 0.5372 & 0.0273 & reject model \\
$\mathrm{SO}_{2}$ & 1 & 0 & 0.6909 & 0.0268 & reject model \\
\hline
\end{tabular}

From Table 3 we reject the GEVL model for $\mathrm{NO}, \mathrm{SO}_{2}$ and accept this model for $\mathrm{NO}_{2}$ pollutant (see Figure 1). Also, we reject the GPDL model for all pollutant, so we applied the Box-Cox GEVL for block maximum data and peak over threshold data. The estimate parameter, AIC, BIC and Kolmogorov-Smirnov test for the Box-GEVL model are summarized in Table 4 and 5. Table 5 show that this model is accepted for extreme data and peak over threshold data (see Figure 2-7). 


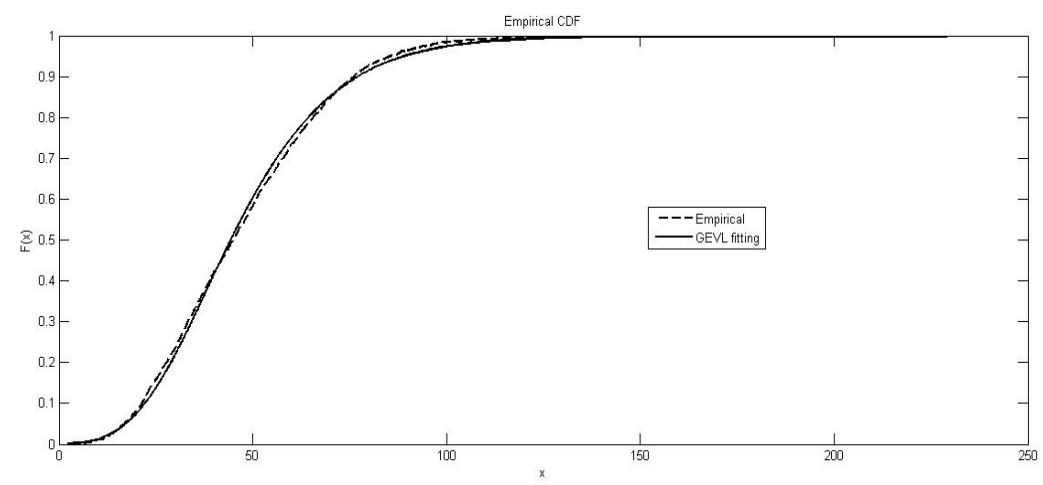

Figure 1: The GEVL model for daily maximum of $\mathrm{NO}_{2}$ concentration for Barking Dagenham station

Table 4: The MLE results, including parameter estimates, (AIC) and (BIC)

\begin{tabular}{||c|c|c|c|c|c|c||}
\hline \multicolumn{6}{|c|}{ Estimation parameter for maximum data by using Box-Cox GEVL. } \\
\hline Pollutant & $\gamma$ & $\sigma$ & $\mu$ & $\lambda$ & AIC & BIC \\
\hline $\mathrm{NO}$ & 0.13 & 6.6 & 6.63 & 0.985 & 1005.6 & 20021.92 \\
\hline $\mathrm{NO}_{2}$ & -0.04 & 19 & 38 & 0.999 & 8952.09 & 17908.6 \\
\hline $\mathrm{SO}_{2}$ & 0.187 & 1.79 & 2.7 & 0.996 & 3790.51 & 8595.58 \\
\hline Estimation parameter for peak over threshold data by using Box-Cox GEVL \\
\hline Pollutant & $\gamma$ & $\sigma$ & $\mu$ (thrushold) & $\lambda$ & AIC & BIC \\
\hline $\mathrm{NO}$ & 0.19 & 51.6 & 31.2 & 0.0004 & 2743.74 & 5516.65 \\
\hline $\mathrm{NO}_{2}$ & -0.066 & 20 & 47.417 & 0.01 & 3584.06 & 7319.45 \\
\hline $\mathrm{SO}_{2}$ & 0.1 & 0.826 & 5.1 & 0.0002 & 1241.53 & 2692 \\
\hline
\end{tabular}

Table 5: K-S test

\begin{tabular}{||c|c|c|c|c|c|}
\hline \multicolumn{6}{|c|}{ Fitting parameter for Box-Cox GEVL model for maximum data } \\
\hline Pollutant & $h$ & $p$ & $K$ & $c . v$ & Decision \\
\hline $\mathrm{NO}$ & 0 & 0.7966 & 0.025 & 0.0272 & accepted model \\
$\mathrm{NO}_{2}$ & 0 & 0.0892 & 0.0245 & 0.0273 & accepted model \\
$\mathrm{SO}_{2}$ & 0 & 0.977 & 0.0023 & 0.0268 & accepted model \\
\hline Fitting parameter for Box-Cox GEVL model for peak over thrushold (average). \\
\hline Pollutant & $h$ & $p$ & $K$ & $c . v$ & Decision \\
\hline $\mathrm{NO}$ & 0 & 0.2795 & 0.0340 & 0.0523 & accepted model \\
$\mathrm{NO}_{2}$ & 0 & 0.2746 & 0.0262 & 0.0486 & accepted model \\
$\mathrm{SO}_{2}$ & 0 & 0.51030 & 0.0226 & 0.0480 & accepted model \\
\hline
\end{tabular}


Empirical COF

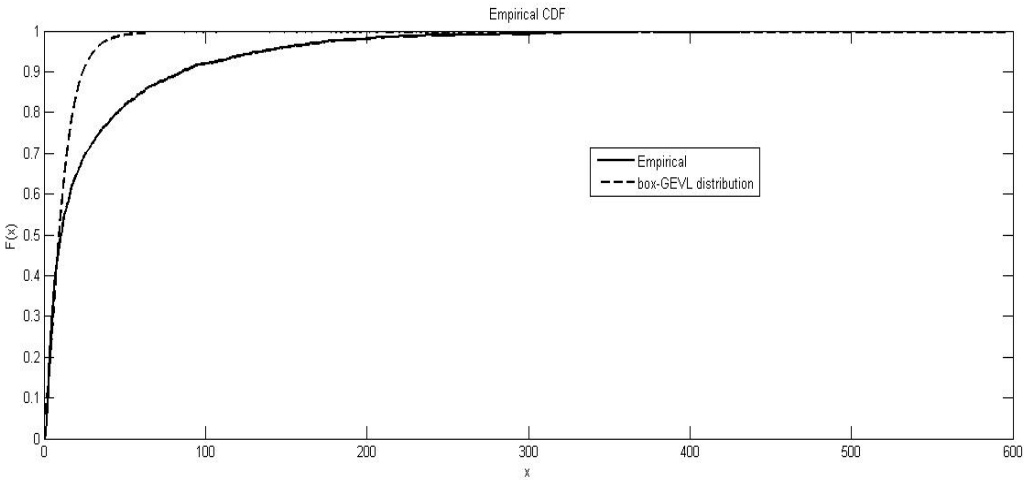

Figure 2: The Box-Cox GEVL for daily maximum of NO concentration for Barking Dagenham station

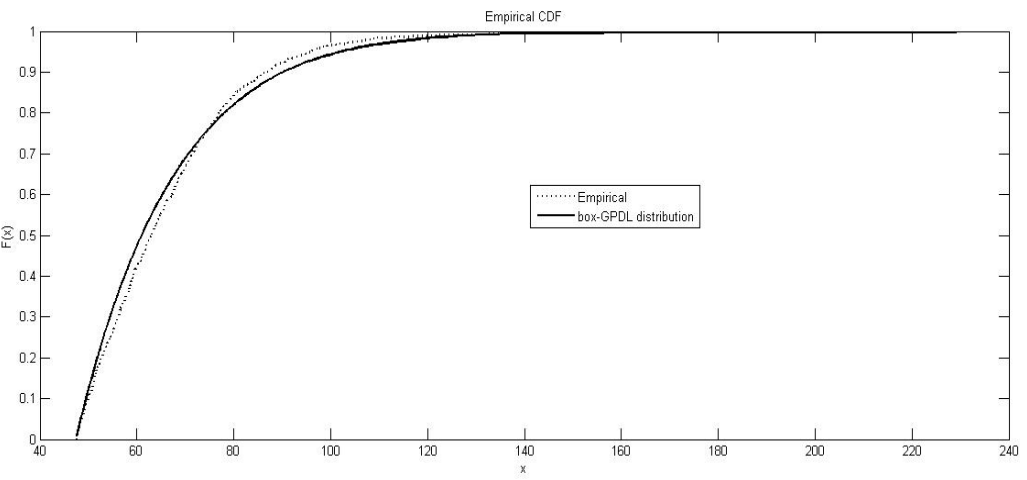

Figure 3: The Box-Cox GEVL for daily maximum of $\mathrm{NO}_{2}$ concentration for Barking Dagenham station

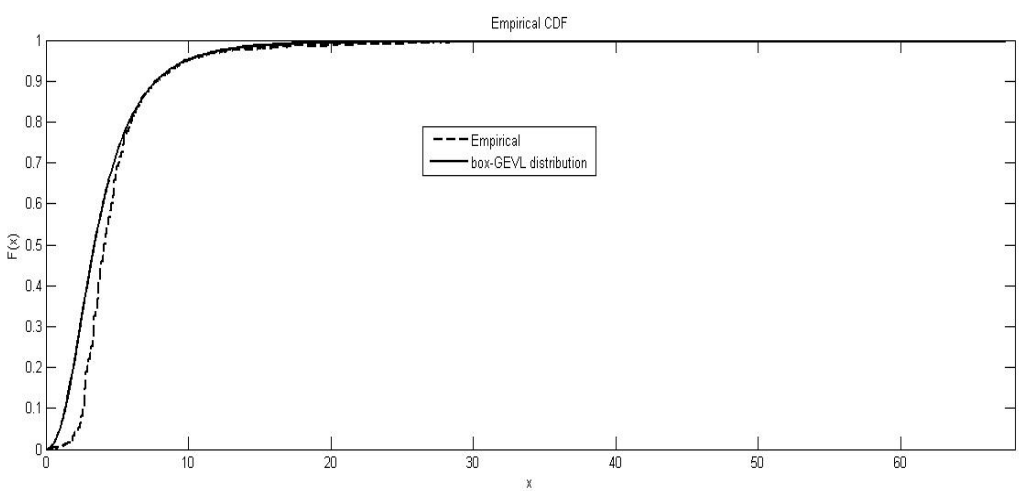

Figure 4: The Box-Cox GEVL for daily maximum of $\mathrm{SO}_{2}$ concentration for Barking Dagenham station 


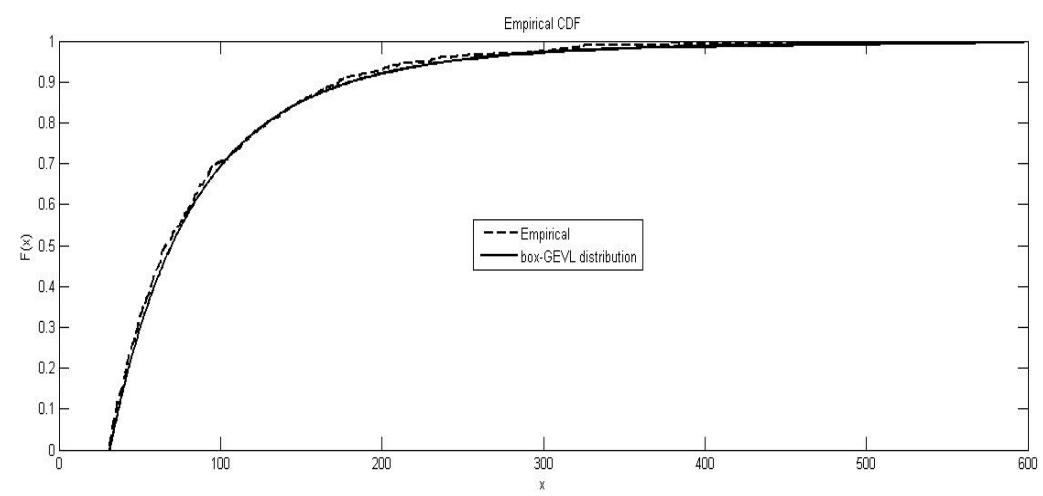

Figure 5: The Box-Cox GEVL for peak over average of NO concentration for Barking Dagenham station

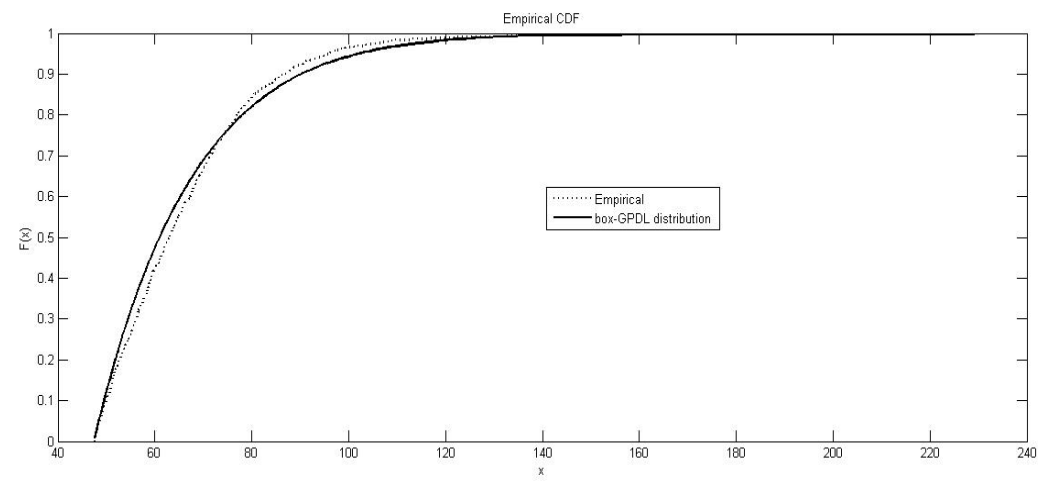

Figure 6: The Box-Cox GEVL for peak over average of $\mathrm{NO}_{2}$ concentration for Barking Dagenham station

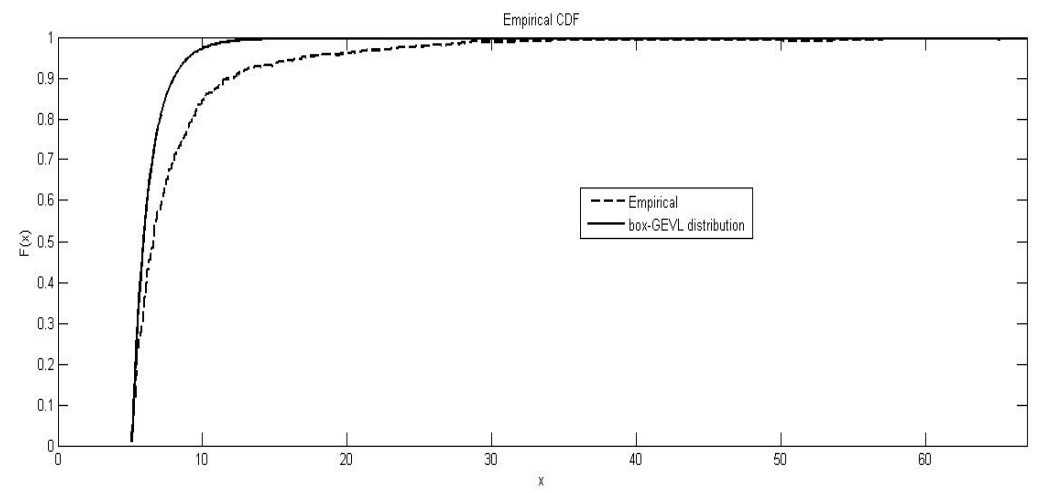

Figure 7: The Box-Cox GEVL for peak over average of $\mathrm{SO}_{2}$ concentration for Barking Dagenham station 
In the second trend, we applied The generlized extreme value distribution under power normalization (GEVP) in Eq. 3.2 for daily maximum data and GPDP model for data peak over threshold (mean). As before the log-likelihood of Box-Cox GEVP is

$$
\ell_{\gamma}^{*}(y ; a, b, \lambda)=k \log b-\sum_{i=1}^{k} \log y_{i}-\lambda \sum_{i=1}^{k}\left(1+\gamma \log a y^{b}\right)^{-\frac{1}{\gamma}}-\left(1+\frac{1}{\gamma}\right) \sum_{i=1}^{k} \log \left(1+\gamma \log a x^{b}\right),
$$

Eq (3.9) gives the log-likelihood for GEVP distribution if $\lambda=1$, and GPDP distribution if $\lambda=0$.

Table 6: The MLE results, including parameter estimates, (AIC) and (BIC)

\begin{tabular}{||c|c|c|c|c|c||}
\hline Estimation parameter for maximum data by using GEVP model \\
\hline Pollutant & $\gamma$ & $\mathrm{a}$ & $\mathrm{b}$ & $\mathrm{AIC}$ & $\mathrm{BIC}$ \\
\hline $\mathrm{NO}$ & -0.1935 & 0.2022 & 0.7993 & 6150.95 & 12320.4 \\
\hline $\mathrm{NO}_{2}$ & -0.2897 & 0.001 & 1.8201 & 9255.05 & 18520.4 \\
\hline $\mathrm{SO}_{2}$ & -0.1744 & 0.0985 & 1.834 & 4690.51 & 9404.53 \\
\hline \multicolumn{6}{|c|}{ Estimation parameter for peak over threshold data } \\
\hline Pollutant & $\gamma$ & $\mathrm{a}$ & $\mathrm{b}$ & $\mathrm{AIC}$ & $\mathrm{BIC}$ \\
\hline $\mathrm{NO}$ & -0.4453 & 0.2338 & 0.5749 & 3090.73 & 6188 \\
\hline $\mathrm{NO}_{2}$ & -0.2624 & 0.00027 & 2.188 & 3970.95 & 7820.68 \\
\hline $\mathrm{SO}_{2}$ & -0.157 & 0.0284 & 2.437 & 1679.19 & 3365.77 \\
\hline
\end{tabular}

The estimation parameter AIC, BIC, and K-S test given in Table 6 and 7, indicates that both GEVP for maximum data and GPDP for peak over thrushold data failed to fit this data. Hence, we applied the suggested model Box-Cox-GEVP for maximum data and truncated data. Results for AIC, BIC and parameter estimate summarized in Table 8. Moreover, the result of the K-S test is given in Table 9, which indicate that the suggested model fits the given data, except $\mathrm{NO}_{2}$ for maximum data (see, Figure 8-12).

Table 7: K-S test

\begin{tabular}{||c|c|c|c|c|c||}
\hline \multicolumn{6}{c}{ Fitting parameter for GEVL } \\
\hline Pollutant & $h$ & $p$ & $K$ & $c . v$ & Decision \\
\hline$N O$ & 1 & 0.00046 & 0.043 & 0.0272 & reject model \\
$N O_{2}$ & 1 & 0 & 0.0656 & 0.0273 & reject model \\
$S O_{2}$ & 1 & 0 & 0.0245 & 0.1063 & reject model \\
\hline \multicolumn{6}{|c|}{ Fitting parameter for GPDL } \\
\hline Pollutant & $h$ & $p$ & $K$ & $c . v$ & Decision \\
\hline$N O$ & 1 & 0 & 0.5454 & 0.0272 & reject model \\
$\mathrm{NO}_{2}$ & 1 & 0 & 0.4613 & 0.0273 & reject model \\
$\mathrm{SO}_{2}$ & 1 & 0 & 0.5505 & 0.0268 & reject model
\end{tabular}$\|$

\section{Discussion and conclusion}

We proposed additional GEV distribution referred to as the Box-Cox GEV distribution and we studied its mathematical properties. We have estimated the parameters of the Box-Cox GEV distribution by the method of maximum likelihood and gave explicit expressions for log likelihood function. We applied two types of models for air pollution in one station of London air quality network called Barking Dagenham. The pollutants $\mathrm{NO}, \mathrm{NO}_{2}$ and $\mathrm{SO}_{2}$ are modeled by GEVL for daily maximum and GPDL for peak over threshold data which are rejected except the pollutant $\mathrm{NO}_{2}$ for maximum data with $\mathrm{AIC}=8952.09$ and $\mathrm{BIC}=17908.6$. The Box-Cox GEVl are applied for maximum data and Peak over threshold data. This model was accepted for $\mathrm{NO}, \mathrm{NO}_{2}$ and $\mathrm{SO}_{2}$ for maximum data with $\mathrm{AIC}=1000.5,8952.09,3790.51$ and $\mathrm{BIC}=20021.92$, 17908.6, 8595.58 respectively, also accepted for threshold data over mean with $\mathrm{AIC}=2743.73,3584.06,1241.5$ and $\mathrm{BIC}=$ $5516.65,7319.45,2692$. The Box-Cox GEVl is more favorable to model the given data of the pollutant except for $\mathrm{NO}_{2}$ which is accepted in Box-Cox GEVl and GEVL for maximum data. However, $\mathrm{NO}_{2}$ had smaller values of AIC and BIC in GEVL 


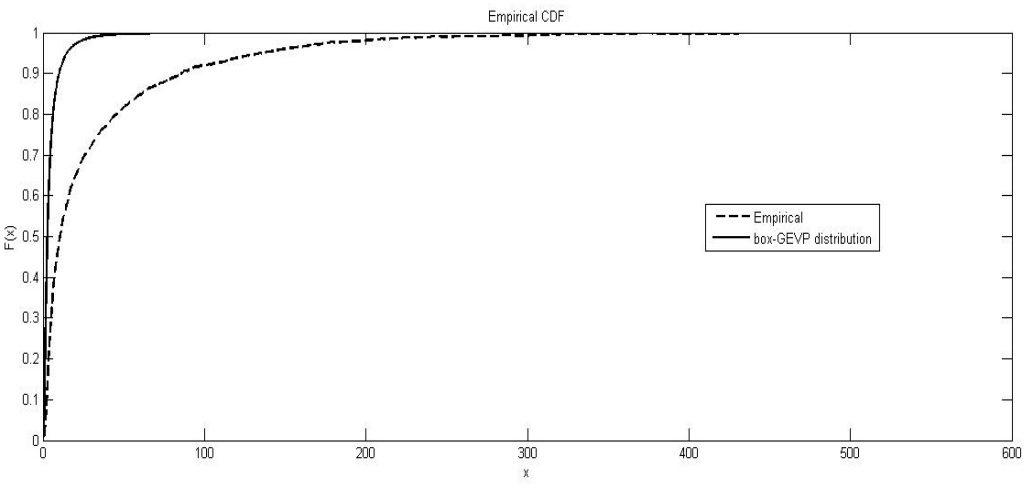

Figure 8: The Box-Cox GEVP model for daily maximum of NO concentration for Barking Dagenham station

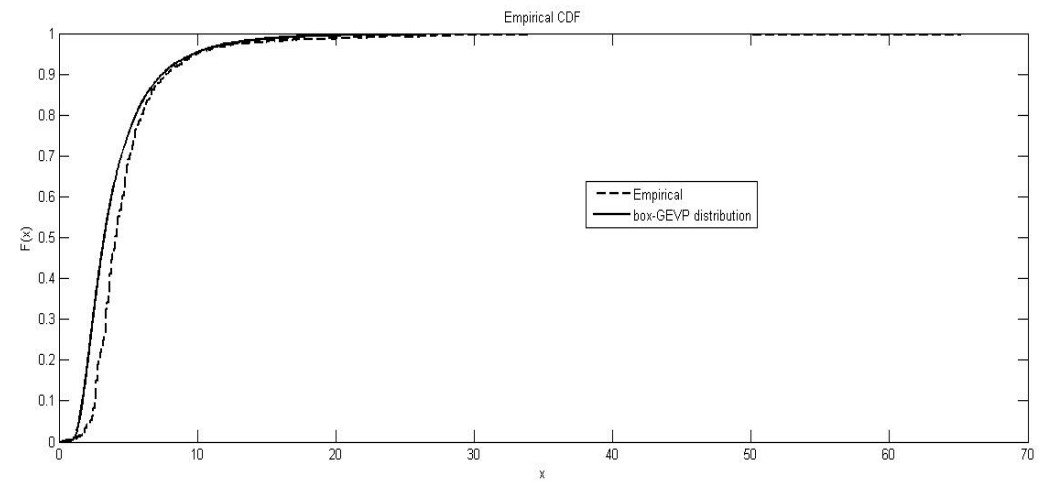

Figure 9: The Box-Cox GEVP model for daily maximum of $\mathrm{SO}_{2}$ concentration for Barking Dagenham station

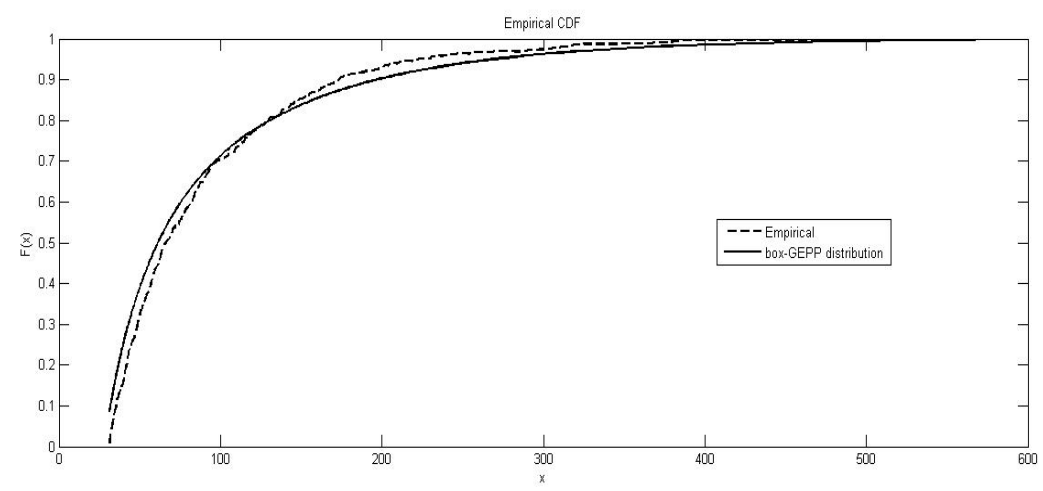

Figure 10: The Box-Cox GEVP model for peak over average of NO concentration for Barking Dagenham station 


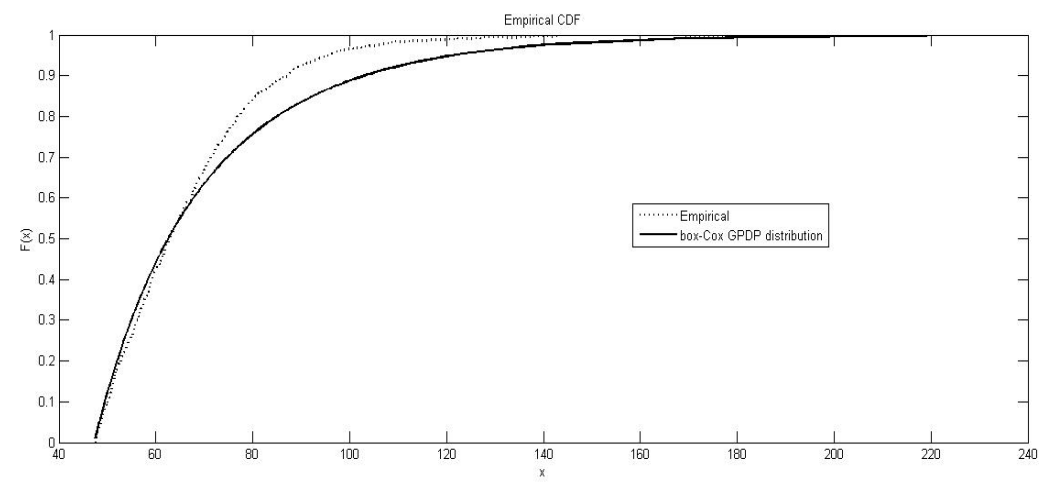

Figure 11: The Box-Cox GEVP model for peak over average of $\mathrm{NO}_{2}$ concentration for Barking Dagenham station

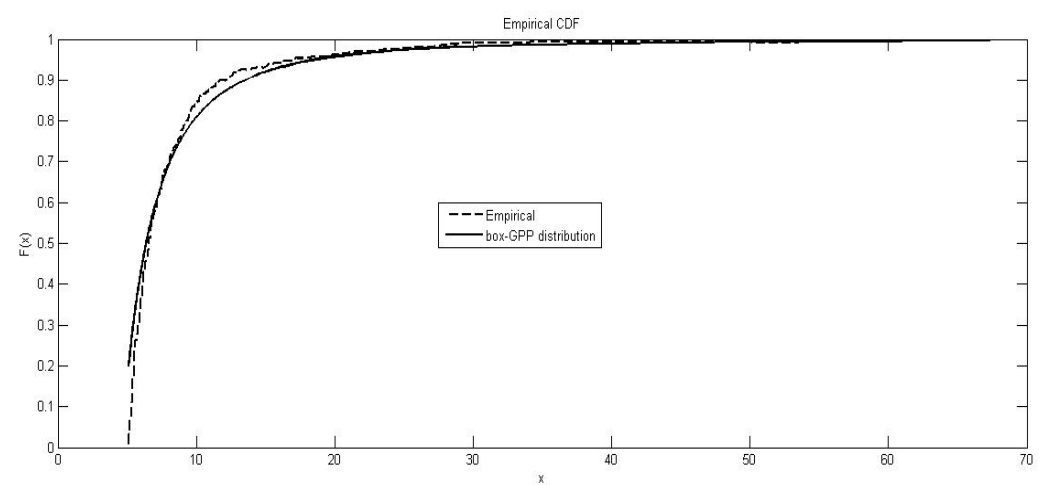

Figure 12: The Box-Cox GEVP model for peak over average of $\mathrm{SO}_{2}$ concentration for Barking Dagenham station 
Table 8: The MLE results, including parameter estimates, (AIC) and (BIC)

\begin{tabular}{||c|c|c|c|c|c|c||}
\hline \multicolumn{6}{c}{ Estimation parameter for maximum data by using Box-Cox GEVP. } \\
\hline Pollutant & $\gamma$ & $\mathrm{a}$ & $\mathrm{b}$ & $\lambda$ & $\mathrm{AIC}$ & BIC \\
\hline $\mathrm{NO}$ & -0.142 & 0.385 & 1.25 & 0.999999 & 5330.96 & 10676.5 \\
\hline $\mathrm{NO}_{2}$ & -0.29 & 0.001429 & 1.84 & 0.999999999999 & 9041.58 & 18094 \\
\hline $\mathrm{SO}_{2}$ & -0.14 & 0.15 & 1.9 & $9.9 * 10^{9}$ & 3796.51 & 78854.2 \\
\hline Estimation parameter for peak over threshold data by using Box-Cox GEVP \\
\hline Pollutant & $\gamma$ & $\mathrm{a}$ & $\mathrm{b}$ & $\lambda$ & $\mathrm{AIC}$ & $\mathrm{BIC}$ \\
\hline $\mathrm{NO}$ & -0.4 & 0.077 & 0.77 & 0.001 & 2609.32 & 5427 \\
\hline $\mathrm{NO}_{2}$ & -0.23 & 0.00029 & 2.2 & 0.012 & 3026.37 & 7064.08 \\
\hline $\mathrm{SO}_{2}$ & -0.011 & 0.04 & 2.11 & 0.009 & 1180.55 & 2570.95 \\
\hline
\end{tabular}

Table 9: K-S test

\begin{tabular}{||c|c|c|c|c|c||}
\hline \multicolumn{6}{c|}{ Fitting parameter for Box-Cox GEVP model for maximum data } \\
\hline Pollutant & $h$ & $p$ & $K$ & $c . v$ & Decision \\
\hline $\mathrm{NO}$ & 0 & 0.9931 & 0.0012 & 0.0272 & accepted model \\
$\mathrm{NO}_{2}$ & 1 & $2.7 * 10^{-6}$ & 0.0564 & 0.0273 & rejected model \\
$\mathrm{SO}_{2}$ & 0 & 0.9406 & 0.0038 & 0.0268 & accepted model \\
\hline Fitting parameter for Box-Cox GEVP & model for peak over thrushold (average). \\
\hline Pollutant & $h$ & $p$ & $K$ & $c . v$ & Decision \\
\hline $\mathrm{NO}$ & 0 & 0.3349 & 0.0315 & 0.0523 & accepted model \\
$\mathrm{NO}_{2}$ & 0 & 0.0657 & 0.0381 & 0.040 & accepted model \\
$\mathrm{SO}_{2}$ & 0 & 0.1136 & 0.0409 & 0.0480 & accepted model \\
\hline
\end{tabular}

which is better. The second trend of study includes the application of the model of GEVP and GPDP for data as before, which are rejected (see Table 7). Therefore, a suggested model Boc-Cox GEVP was applied. This new model was accepted for $\mathrm{NO}$ and $\mathrm{SO}_{2}$ with $\mathrm{AIC}=5330.96,3796.51$ and $\mathrm{BIC}=10676.5,73854.2$ respectively and it was rejected for $\mathrm{NO}_{2}$ for maximum data. Moreover, it was accepted for $\mathrm{NO}, \mathrm{NO}_{2}$ and $\mathrm{SO}_{2}$ with $\mathrm{AIC}=2609.32,3026.37,1180.55$ and $\mathrm{BIC}=5427,7064.08$, 2570.959 for peak over threshold data. It could be concluded that in this study the models from Boc-Cox GEVP is better than Boc-Cox GEVL based on AIC and BIC criteria for pollutant $\mathrm{NO}$ and $\mathrm{SO}_{2}$, while, GEVL model is the best for pollutant $\mathrm{NO}_{2}$.

\section{Acknowledgments}

The authors would like to thank the Editor and the referee for careful reading and comments which greatly improved the paper.

\section{References}

[1] A.F. Jenkinson, The frequency distribution of the annual maximum (or minimum) values of meteorological elements. Q. J. Roy. Meteor. Soc. 87 (1955) 145158.

[2] J.Pickands, Statistical inference using extreme order statistics. Ann. Stat. 3 (1975) 119-131.

[3] T. B. Bali, The generalized extreme value distribution, Econ. Letters 79 (2003) 423-427

[4] D. Nasri-Roudsari, Limit distributions of generalized order statistics under power normalization. Commun. Stat. Theor. Methods 28(6) (1999) 1379-1389.

[5] H. M. Barakat, E. M. Nigm, and O. M. Khaled, Statistical modeling of extremes under linear and power normalizations with applications to air pollution. Kuwait J. Sci. 41 (2014), 1-19.

[6] H. M. Barakat, E. M. Nigm, and O. M. Khaled, Extreme Value Modeling under Power Normalization, Appl. Math. Modell. 37 (2013), 10162-10169 
[7] H. M. Barakat, A. R. Omar, and O. M. Khaled, A new flexible extreme value model for modeling the extreme value data, with an application to environmental data, SATAT. PROBABIL. IETT. (2017), doi.org/10.1016/j.spl.2017.07.002

[8] G. Box, D. Cox, An analysis of transformations. J. R. Stat Soc. B. (1964) 211264.

[9] NR. Mohan, S. Ravi Max domains of attraction of univariate and multivariate p-max stable laws. Theor. Probability Appl. 37 (1992) 632-643.

[10] E. Pantcheva, Limit theorems for extreme order statistics under nonlinear normalization. Lect. Not. Math. 1155 (1984) 284-309.

[11] U. R. Subramanya, On max domains of attraction of univariate p-max stable laws. Stat. Probability. Lett., 19 (1994) 271-279.

[12] G. Christoph, and M. Falk, A note on domains ofattraction of p-max stable laws. Stat. Probability. Lett. 28 (1996) 279-284.

[13] G. Fuller, P. Johnson, A. Cue, Air quality in London 2001:the ninth report of the London Air Quality Network. London, Environmental Research Group, Kings College London, 2003.

[14] C. David Carslaw, D. Sean Beevers and Gary Fuller .An empirical approach for the prediction of annual mean nitrogen dioxide concentrations in London,Atom. Environ. 35 (2001) 1505-1515

[15] R. Maheswaran, T. Pearson, SD. Beevers ,MJ. Campbell, CD. Wolfe Air Pollution and Subtypes, Severity and Vulnerability to IschemicStrokeA Population Based Case-Crossover Study. Plos One 11(6) (2016) e0158556. doi: 10.1371/journal.pone.0158556.: 